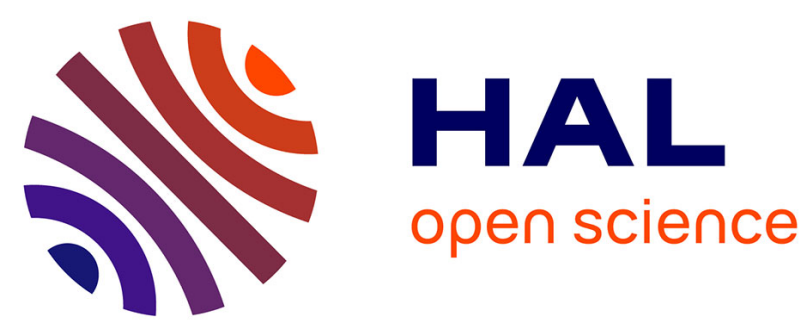

\title{
Intermittent turbulence and copepod dynamics: increase in encounter rates through preferential concentration
}

\author{
François G Schmitt, Laurent Seuront
}

\section{To cite this version:}

François G Schmitt, Laurent Seuront. Intermittent turbulence and copepod dynamics: increase in encounter rates through preferential concentration. Journal of Marine Systems, 2008, 70 (3-4), pp.263272. 10.1016/j.jmarsys.2007.01.008 . hal-00263401

\section{HAL Id: hal-00263401 \\ https://hal.science/hal-00263401}

Submitted on 12 Mar 2008

HAL is a multi-disciplinary open access archive for the deposit and dissemination of scientific research documents, whether they are published or not. The documents may come from teaching and research institutions in France or abroad, or from public or private research centers.
L'archive ouverte pluridisciplinaire HAL, est destinée au dépôt et à la diffusion de documents scientifiques de niveau recherche, publiés ou non, émanant des établissements d'enseignement et de recherche français ou étrangers, des laboratoires publics ou privés. 


\title{
Intermittent turbulence and copepod dynamics: increase in encounter rates through preferential concentration
}

\author{
François G. Schmitt ${ }^{a, *, 1}$, Laurent Seuront ${ }^{\mathrm{a}, \mathrm{b}}$ \\ ${ }^{a}$ CNRS, FRE 2816 ELICO, Wimereux Marine Station, Université des Sciences et \\ Technologies de Lille - Lille 1, 28 av. Foch, 62930 Wimereux, France \\ Fax +33-321992901 \\ ${ }^{\mathrm{b}}$ School of Biological Sciences, Flinders University, GPO Box 2100, Adelaide \\ 5001, South Australia
}

\begin{abstract}
Turbulence has a strong influence on plankton contact rate, which is a crucial parameter for plankton ecology. In the field of particle-turbulence interactions, it is now well known that fully developed turbulence does not always homogenise particle distributions, but instead creates, in some well-defined conditions, preferential concentrations of heavy particles. Many studies have considered the influence of this type of preferential concentration on particle contact rate. We consider here the possible application of these results to copepods, assuming that some results obtained for heavy particles are still valid for light particles. Using parameter values representative of copepod species in coastal waters and open ocean, we numerically estimate the possible enhancement of copepod contact rates due to the preferential concentration by turbulence. The assessment is done by using data from a laboratory experiment: we find from the trajectory analysis of small neutrally buoyant particles, that the preferential concentration effect increases the contact rate up to $140 \%$. We argue that this effect may be more pronounced for higher Reynolds numbers, and may have important ecological applications.
\end{abstract}

Key words: Turbulence, copepods, preferential concentration, scaling, contact rate

* Corresponding author.

1 francois.schmitt@univ-lille1.fr 


\section{Introduction}

Turbulence is now widely recognized to influence dispersal, feeding and reproduction of copepods. Two of the major aspects of copepod dynamics involve contact rates between particles in turbulent flows: predator-prey encounter rates, and mating encounter rates. In copepod ecology, a large body of literature has considered encounter rate enhancement through remote detection ability and behavioural activity. This includes chemoreception and mechanoreception (Buskey, 1984; Weissburg et al., 1998), prey movement detection (Visser, 2001; Jiang et al., 2002), feeding currents (Marrassé et al., 1990) and swimming behaviour (Gerritsen and Strickler, 1977; Rothschild and Osborn, 1988; Evans, 1989; Hill et al., 1992; Visser and MacKenzie, 1998; Titelman, 2001). The effect of the behaviour is, however, only effective within a limited distance between organisms. The encounter rate can thus be modelled as a combination of two independent, though complementary, physically- and biologically-driven processes. First, turbulence brings two particles (either a predator and its prey, or two mates) below a threshold detection distance. Then, when the detection distance is reached, behavioural processes are triggered, leading to attempts to capture the encountered particles (i.e. a prey or a mate). While we only consider the effect of turbulence on copepod encounter rates, without considering the subsequent behavioural processes, the behavioural contribution would further enhance the encounter rates (see e.g. Kiørboe and Saiz (1995); Seuront et al. (2001)). In the plankton ecology literature, turbulence is implicitly assumed to homogenise particle distributions at small scales. In contrast, in the community of turbulence physics, turbulence is widely acknowledged to increase inhomogeneity at small scales, which is referred to as the intermittency effect (Frisch, 1995).

Concerning particles, three types of effect are expected, depending on the inertia of these particles (their Stokes number, considered in the next section). Zero inertia particles behave as passive tracers, purely passively advected by the flow, whereas heavy particles (infinite inertia) have their own trajectories and are not influenced by turbulence. In contrast, intermediate inertia particles are only influenced by some portions of turbulent motions as they cannot be influenced by the largest vortical motions due to their finite inertia (Wang et al., 2000). As a consequence, particles denser than then fluid cannot stay in high vorticity regions, and have larger concentrations in low vorticity and high strain rate regions. The result is known as the phenomenon of preferential concentration of heavy particles due to turbulence (Maxey, 1987; Squires and Eaton, 1991; Wang and Maxey, 1993; Fessler et al., 1994). This "inertia difference" effect can also be interpreted as being due to the difference in inertia of a particle and the fluid it displaces, for particles having a density larger than the density of the fluid (density ratio $>1$ ). However, particles may tend to migrate towards the center of vortices because a small difference in 
acceleration can be amplified when the particle approach a hyperbolic point in the fluid flow (Babiano et al., 2000). This occurs at all density ratios, but is most evident when the density ratio is close to 1 . This has only been shown in models, and in the following we will not consider this case and focus on density ratio larger than 1, although in copepod applications this ratio is close to 1 .

The effects of preferential concentration have mainly been studied on the coagulation of colloidal particles, with aerosols, sprays and cloud physics applications in mind (Brunk et al., 1988; Vaillancourt and Yau, 2000; Wang et al., 2000; Zhou et al., 2001; Falkovich et al., 2002). Its application to copepod ecology has been, to our knowledge, only considered in one paper (Squires and Yamazaki, 1995), using Direct Numerical Simulation (DNS) of flow equations (see also a discussion by Jimenez (Jimenez , 1997)). This paper showed that preferential concentration of particles presenting material characteristics close to those encountered in marine environments can occur, with peak particle densities ranging from 10 to 60 times the mean value. This early work was published before the precise effect of preferential concentration on particle contact rates was extensively studied in a series of papers by several teams of researchers (Sundaram and Collins, 1997; Zhou et al., 1998; Wang et al., 1998, 2000; Reade and Collins, 2000; Collins and Keswani, 2004). We discuss here the application of these results to copepods, and show its potential importance for the estimation of copepod encounter rates. In section 2, we review the most salient results of the particle turbulence literature concerning preferential concentration and encounter rates. In section 3 these results are considered in the framework of copepod ecology. In particular, Stokes numbers, characterizing the inertia of particles relative to local turbulence properties, are estimated for characteristic flow and copepod properties, and the relevance of the previous inertial particle framework to copepods is discussed. We also investigate the possible effects on radial distribution function and enhancement of copepod encounter rates. An experimental database of polystyrene particle dispersion by turbulence is analyzed as an example of preferential concentration of buoyancy free particles. Finally, we discuss the potential importance of this phenomenon for the estimation of encounter rates in aquatic ecology.

\section{Preferential concentration in turbulence and contact rate}

\subsection{Contact rate in homogeneous prey fields}

The encounter rate and its relevant estimation in various experimental conditions is a critical issue in plankton ecology. Models of encounter rates have been originally proposed for motile predators and preys in non-turbulent conditions. For a predator moving at a constant velocity $V$ in an homogeneous 
field of non-motile prey (concentration $n$ ) without turbulence, the encounter rate is written as:

$$
Z=n \pi R^{2} V
$$

where $Z$ is the number of encounters per unit volume per unit time, $R$ is the encounter, or perceptive, radius of the predator. This expression becomes more complex when the prey have a non-zero velocity. Considering a homogeneous field of prey moving in random directions at a velocity $U$, and a predator moving at a constant velocity $V$ (we consider here the case $V \geq U$ ) Eq. (1) becomes (Gerritsen and Strickler, 1977):

$$
Z=n \frac{\pi R^{2}}{3}\left(\frac{U^{2}+3 V^{2}}{V}\right)
$$

To take turbulenceinto account, Eq. (2) has been modified by Rothschild and Osborn (1988), with further contributions from Evans (1989) and Visser and MacKenzie (1998) (see a review in Lewis and Pedley (2000)). For zero velocity predator and prey $(U=V=0)$ in a homogeneous turbulence medium, the formulae given by Rothschild and Osborn (1988) involves only turbulent velocity and recovers the standard expression of particle encounter rates in turbulence (Saffman and Turner, 1956; Delichatsios and Probstein, 1975; Hill et al., 1992; Wang et al., 2000):

$$
Z=n \pi R^{2}<w_{r}(R)>
$$

where $<$. $>$ means ensemble average, $w_{r}(R)=|W(x+R)-W(x)|$ is the radial turbulent velocity between two particles separated by a distance $R$, and $\left\langle w_{r}(R)\right\rangle$ is the mean amplitude of the relative radial velocity (the subscript "r" is for radial). This expression is analogous to Equation (1), with the predator constant velocity replaced by the turbulent term $\left\langle w_{r}(R)>\right.$.

\subsection{Modification of the contact rate in the case of preferential concentration of particles}

In fully developed turbulence, it has been recognized since Maxey (1987) that inertial particles are inhomogeneously distributed; see also Squires and Eaton (1991), Wang and Maxey (1993), and Fessler et al. (1994). Due to their inertia, spherical particles of diameter $\sigma$ are ejected from highly vortical regions, and the resulting local particle concentration is higher than the average, leading to a correction term for the encounter rate expression. This leads to the following expression (Sundaram and Collins, 1997; Wang et al., 1998; Reade and Collins, 2000; Collins and Keswani, 2004):

$$
Z=n \pi \sigma^{2} g(\sigma)<w_{r}(\sigma)>
$$


where the encounter (i.e. contact) between particles occurs at a distance $r=\sigma$. The term $\left\langle w_{r}(\sigma)>\right.$ expresses the radial relative velocity between particles, estimated at contact and is the dynamical aspect of the encounter rate. In Equation (4), the important new term is the correction factor $g(\sigma)$ related to preferential concentration. The function $g(r)$ is the radial distribution function, also called the pair correlation function. It measures the level of particle pair accumulation with interparticle distance $r$, and thus directly represents the accumulation effect. This is a standard function in statistical mechanics (Pathria, 1972), widely used in various fields (Bernal et al., 1962; Bishop and Bruin, 1984; Lock and Chiu, 1994) to characterize the distribution of particles. An introduction to the concept and its implementation can be found in Younge et al. (2004). It may be written as the probability of finding a particle in a given volume $d V$ at distance $r$, normalized by the probability of finding a particle in $d V$ if the particles were uniformly distributed. Hence it is also a local measure of how uniform the particle distribution is, it is close to 1 for homogeneous distributions and is larger for inhomogeneous distributions.

This function depends on the Reynolds number and on the Stokes number St. The Stokes number is the non-dimensional ratio of an inertial characteristic time scale $\tau_{p}$ (also called the aerodynamic response time, see Fessler et al. (1994)), to a turbulent characteristic time (the Kolmogorov time scale) $\tau_{\eta}$. This gives (Reade and Collins, 2000):

$$
S t=\frac{\tau_{p}}{\tau_{\eta}}=C_{p}\left(\frac{\sigma}{\eta}\right)^{2}
$$

with the constant:

$$
C_{p}=\frac{\beta}{18}
$$

where $\beta=\rho_{p} / \rho$ is the ratio of the particle density to the fluid density, and

$$
\eta=\left(\frac{\nu^{3}}{\epsilon}\right)^{1 / 4}
$$

is the Kolmogorov length scale. The latter can also be written using the Reynolds number and the integral length scale $L$ as $\eta=L R e^{-3 / 4}$. The Stokes number characterizes the inertia of particles and is one of the fundamental parameters characterizing particle-turbulence interactions: for $S t \ll 1$, particles follow passively the flow, whereas for $S t \gg 1$, large inertia particles are not influenced by turbulence and follow their own trajectories.

Let us consider the $S t$ dependence, rewriting the function $g$ as $g(r, S t)$. The function $G(S t)=g(\sigma, S t)$ has been studied numerically through DNS; $r=\sigma$ corresponds to a contact at particle diameter distance (Sundaram and Collins, 1997; Wang et al., 1998, 2000; Reade and Collins, 2000). We reproduce in Figure 1 one of the typical curves (adapted from Wang et al. (2000)), showing 
$G(S t)$ as a function of the Stokes number $S t$ for three different values of the microscale Reynolds number $R_{\lambda}$. In this example, $G(S t)$ is maximal for $S t$ values close to 1 . As previous studies found this function maximal for $S t$ close to 0.4 (Sundaram and Collins, 1997) or 0.8 (Reade and Collins, 2000), it is justified to consider here that $G(S t)$ is maximal for values ranging from 0.4 to 1 . Furthermore, the maximal value obtained for $G(S t)$ can be as high as 12 to 200, depending on the simulation considered using various microscale Reynolds numbers and particle diameters. The encounter rate enhancement factor due to preferential concentration thus ranges between 1 to 2 orders of magnitude.

The function $g(r, S t)$ has also been studied numerically for fixed $S t$ values (Reade and Collins, 2000; Collins and Keswani, 2004), in this case we keep below the notation $g(r)$. It has been shown that the residual radial distribution function $h(r)=g(r)-1$ has a power-law $r$ dependence, of the form (Reade and Collins, 2000; Collins and Keswani, 2004):

$$
h(r)=c_{0}\left(\frac{r}{\eta}\right)^{-c_{1}}
$$

with $c_{0}=1.75 \pm 0.1$ and $c_{1}=0.65 \pm 0.1$ for values of $S t$ between 0.7 and 1 (Collins and Keswani, 2004). This power-law fit has been shown to be very close to data for $r / \eta$ values between $5 \times 10^{-3}$ and 1 (Collins and Keswani, 2004), but it is still approximately valid for $r / \eta=2$ (see Reade and Collins (2000)). Rewriting Equation (8) for a distance $r>\sigma$ and for $r=\sigma$ leads to:

$$
h(r)=h(\sigma)\left(\frac{r}{\sigma}\right)^{-c_{1}}
$$

This provides a correction factor for the radial distribution function when the encounter distance is larger than the particle diameter. This will be particularly useful for the copepod encounter rate, as their perceptive distance increases their effective size.

\section{Application to copepods}

\subsection{Copepod Stokes number and applicability of DNS results}

For the previous framework to be applicable to copepods, we consider a hypothetical non-motile copepod population uniform in size. Let us consider the Stokes number for copepods. First, the constant $C_{p}$ (Equation (5)) is estimated as:

$$
C_{p}=5.7 \pm 0.1 \times 10^{-2}
$$




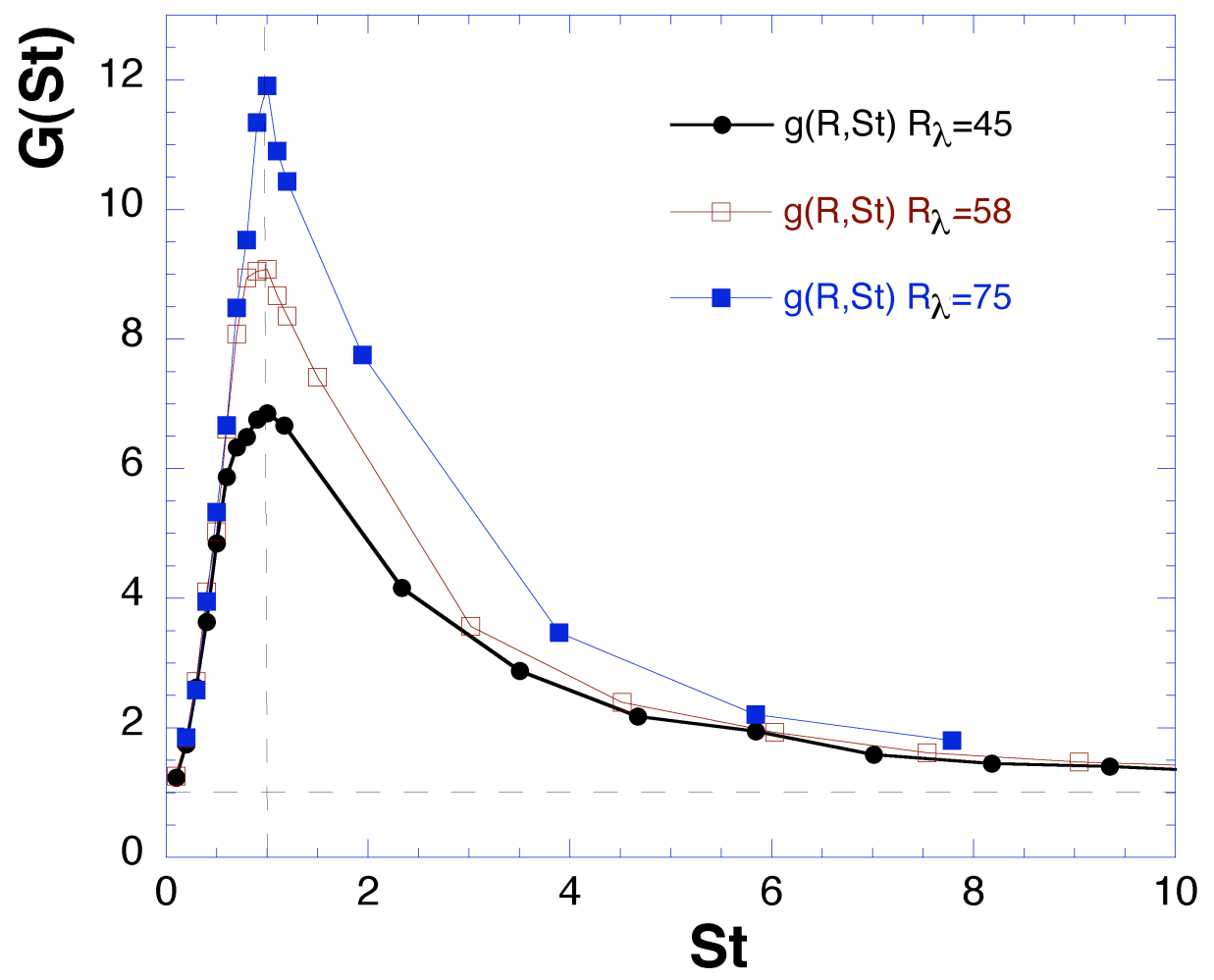

Fig. 1. Representation of the radial distribution function at contact $G(S t)$ versus the Stokes numbers, and for various microscale Reynolds numbers $R_{\lambda}$. Data from DNS simulation kindly provided by L. P. Wang (Wang et al., 2000).

considering a copepod density $\rho_{p}$ bounded between 1.02 to $1.08 \times 10^{3} \mathrm{~kg} / \mathrm{m}^{3}$ (McCave, 1984), the sea water density of $\rho=1.027 \times 10^{3} \mathrm{~kg} / \mathrm{m}^{3}$, and the subsequent ratio $\beta=\rho_{p} / \rho$ close to 1 . Considering Eq.(5) and writing the optimal Stokes number as $S t=0.7 \pm 0.3$, the optimal copepod diameter, relative to the Kolmogorov turbulent scale $\eta$ is:

$$
\frac{\sigma}{\eta}=3.5 \pm 1
$$

As a consequence, to have a Stokes number bounded between 0.4 and 1, as a copepod needs to be larger than the Kolmogorov scale. The studies discussed in the previous section referred to inertial particles having a much larger density ratio than copepods (i.e. $\beta \approx 800$ for rain droplets, and $\beta \approx 800$ to 1500 for aerosol particles), so that the particle diameter could be much smaller than the Kolmogorov scale.

Since we consider here particles having a size larger than the Kolmogorov scale, there is another alternative for the choice of a relevant Stokes number associated to such particles. We can consider that larger particles distort the turbulence at scales below the particles' diameter: they are not sensitive to velocity fluctuations at scales below $\sigma$, and hence a new Stokes number 
shall be considered. The previous Stokes number may be called "Kolmogorov Stokes number" (Loth, 2000), being a ratio of particle relaxation time to the Kolmogorov time scale. A new Stokes number may be considered as relevant if one considers the time scale associated to eddies of spatial scale $\sigma$. It may be written:

$$
S t_{\sigma}=\frac{\tau_{p}}{\tau_{\sigma}}
$$

where $\tau_{\sigma}=\tau_{\eta}(\sigma / \eta)^{2 / 3}$ is due to dimensional arguments in the inertial range, for scales $\sigma>\eta$. This gives (using Eq. (5)):

$$
S t_{\sigma}=S t_{\eta}\left(\frac{\sigma}{\eta}\right)^{-2 / 3}=C_{p}\left(\frac{\sigma}{\eta}\right)^{4 / 3}
$$

In this case the previous expression for the optimal copepod diameter associated to the optimal Stokes number of $S t=0.7 \pm 0.3$ is modified, and becomes:

$$
\frac{\sigma}{\eta}=6.4 \pm 2
$$

which is still a distance larger than the Kolmogorov scale, and is about twice the previous optimal diameter.

Another important difference with the framework presented in section 2 is the existence of an encounter radius, $R$, for zooplankters $(R>\sigma)$; they can grab an incoming mate or particle before the latter is in contact which corresponds to an increase of their effective size. This property has several implications on the framework presented above. First, Eq. (4) has to be modified, replacing the particle diameter $\sigma$ by the encounter radius $R$, except for the Stokes number which is purely driven by the absolute size of the copepod. The resulting copepod contact rate with preferential concentration is then the following, using Eq. (4):

$$
Z=n \pi R^{2} g(R)<w_{r}(R)>
$$

where $g(R)$ is given by Eq. (9):

$$
g(R)=1+(g(\sigma)-1)\left(\frac{R}{\sigma}\right)^{-c_{1}}
$$

Considering a typical value for copepods of $R / \sigma=2$ (Lenz and Yen (1993) find $R / \sigma \approx 1$, but other studies experimentally find values close to 3 (Bagoien and Kiorboe, 2005; Doall et al., 1998)) and $c_{1}=0.65$, the radial distribution function at contact $g(\sigma, S t)=G(S t)$ is multiplied by a factor of about 0.6 , so that $g(R)$ still reaches high values. This shows that the effect of preferential concentration may be important for copepods: copepods with the adequate size will have a much higher contact rate than others with a different size. The optimal size is given by Eq.(11) and depends on the Kolmogorov scale. The contact rate is given by Eq.(15), with a correction factor relative to the classical expression (Eq.(3)), provided in Eq.(16). 
We note here that the numerical studies discussed above are based on direct numerical simulations of the Navier Stokes equations, together with the use of Maxey-Riley-Gatignol equation for particle dynamics (Maxey and Riley, 1983; Gatignol, 1983), and are only valid for particles diameters much smaller than the Kolmmogorov scale (see (Michaelides, 2003) for a review). This means that these numerical results cannot be applied to simulate the dynamics of copepods larger than the Kolmogorov scale. This is also an implicit weakness in the approach of Squires and Yamazaki (1995). The equation for such large particles in turbulent flow are still unknown, even for spherical particles. For numerical studies of the dynamics of large particles (typically of the size of the smallest eddies) one needs to explicitly take into account the shape of the particles (Pan and Banerjee, 1997). Nevertheless, this does not invalidate the equations for contact rates, which are still valid (Eqs.(4) and (15)) since they have been obtained analytically using very general assumptions involving an appropriate statistical average of the collision operator over all possible particle locations and velocities (Sundaram and Collins, 1997). The numerical values of $G(S t)$ and $g(r)$ have been obtained numerically, and need to be confirmed by experiments with particles having a density and size close to the ones of copepods. An experimental study conducted in this specific framework is presented in the next section.

\subsection{Application to experimental dispersion data}

We consider here the experimental data presented in Mann et al. (1999) and Ott and Mann (2000). A water tank of inner dimension $320 \times 320 \times 450 \mathrm{~mm}^{3}$, with two oscillating grids at the top and the bottom, was used to generate a flow with no mean velocity and approximately homogeneous and isotropic turbulence at the central region. The flow was seeded with polystyrene particles with diameters in the range $500-600 \mu \mathrm{m}$. A heat treatment was applied to the particles in order to adjust their density to the water density. The resulting density was also very close to that of the copepods. Video images were taken by four synchronized CCD cameras. A light source was provided through a Xenon discharge tube synchronised with the cameras, with flashes at a rate of 25 flashes per second. Raw images were treated using an image analysis software, in order to provide particle positions in time; recorded positions were restricted to a spatial region of $140 \times 140 \times 120 \mathrm{~mm}^{3}$. About 700 to 1000 particles were used for each experiment. Among the available databases, we have considered here the one corresponding to the largest Reynolds number. The Taylor microscale Reynolds number is $R_{\lambda}=104$, the dissipation $\epsilon=2.7910^{-4} \mathrm{~m}^{2} \mathrm{~s}^{-3}$, the kinematic viscosity of the water is $\nu=0.89 \quad 10^{-6}$ $\mathrm{m}^{2} / \mathrm{s}$. Using Eq. (7) giving the Kolmogorov length scale $\eta$ and the analogous expression for the Kolmogorov time scale $\tau_{\eta}=(\nu / \epsilon)^{1 / 2}$, one obtains $\eta=0.22 \mathrm{~mm}$ and $\tau_{\eta}=0.056 \mathrm{~s}$. Using Eq.(5) and $C_{p}$ given by Eq.(10), one 
obtains a Stokes number for these particles in this flow of $S t=0.4$. On the other hand, if one considers the Stokes number at scale $\sigma$ (Eq. (13)), one obtains $S t=0.2$, which is slightly outside the possible range of the preferential concentration effect.

While Ott and Mann (2000) and Mann et al. (2005) have considered dispersion properties using these particles, we focus here on the preferential concentration effect. Since the particle and flow characteristics are close to those corresponding to the copepod framework: the value of $\beta$ is close to 1 , and the Stokes number is close to the range $(0.4,1)$ corresponding to the preferential concentration effect, according to the numerical results reported above. The Stokes number values $S t=0.2-0.4$ are at the lower end of the range of values corresponding to preferential concentrations. Additional experiments are needed to reach a Stokes number close to 1 . This database nevertheless shows a slight preferential concentration effect. A $2 \mathrm{D}$ projection of a $3 \mathrm{D}$ snapshot (Fig. 2) shows a slight accumulation of particles. This visual impression is quantitatively confirmed by the radial distribution function $g(r)$, estimated from 3D positions of particles, and as an ensemble average over all the particles and for all the successive discrete times available. There are 1438 times, corresponding to a record duration of about $57 \mathrm{~s}$, and there is an average number of 774 particles for each time. The number of particles taken into account in the estimation of $g(r)$ is hence $1438 \times 774$. There is a mean particle density over the whole data set of 0.13 particles per $\mathrm{cm}^{3}$. The radial distribution function is estimated as described in Younge et al. (2004): bins were chosen going from the minimal interparticle distance $\left(d_{\min } / \eta=2.7\right)$ to a maximum distance of $d_{\max } / \eta=347$ with a size exponentially increasing. 37 bins have been taken, with a width going from $1.14 \eta$ to $17 \eta$. The number of particle couples in each bin is variable, with a minimum value of $810^{4}$. The result is shown in Figure 3. The function peaks to a value of about 2.4 for small distance while the optimal value corresponds to a distance of twice the diameter of particles, taken here as $\sigma=0.55 \mu \mathrm{m}$. This maximum value of $g(r)$ is compatible with the numerical results, taking into account the fact that the Stokes number here is not the optimal one and that the distances considered are larger than the ones considered for numerical simulations.

This shows directly that preferential concentration is possible for light and large particles, having properties (density, size) compatible with copepods. We may mention here a discussion by Jimenez (1997) where he showed, using a simplified equation of motion for a particle, that when particle density and fluid density are close to each other, preferential concentration cannot occur. However, this result may not be valid when the full particle equation is taken into account, as indicated by the experimental results presented here. Since the radial distribution function is a multiplicative factor in the encounter rate, the value close to 2.4 that was found here corresponds to an increase of $140 \%$ of the encounter rate, relative to the standard expression. This corrective effect 
is thus already very important even if the Stokes number is not optimal. It can then realistically be expected that the corrective factor reaches much higher values for Stokes numbers closer to 1, and for larger Reynolds numbers.

The optimal Stokes number for the preferential concentration effect, for particles slightly larger than the Kolmogorov scale, and of density close to the density of the fluid, is not known experimentally nor numerically, since most numerical and experimental papers on this topic have focused on heavy and smaller particles. We have seen here that the preferential concentration effect exists for larger particles but more thorough analysis is needed to determine the optimal Stokes number. In future work, we plan to estimate the effect of the Reynolds number on $g(r)$ for the same type of flow where increasing the Reynolds number decreases the Kolmogorov scale, and hence increases the Stokes number, for the same particles.

We also mention here the possible influence of sedimentation on particle concentration. Indeed, using DNS data Wang and Maxey (Wang and Maxey, 1993) have found an increase of the mean settling velocity of particles when their density is larger than the density of the fluid, due to a preferential sweeping effect. One may also find more recent studies on turbulence increasing the average settling velocity using numerical (Bosse et al., 2006) as well as experimental data (Aliseda et al., 2002; Ruiz et al., 2004). In their experimental study of the average settling velocity of phytoplankton cells, Ruiz et al. (2004) have obtained a very small average settling velocity. Thus, this phenomenon is not expected to have an influence over small time scales, as considered in the experiment outlined in the present paper. Furthermore, Mann et al. (1999) have estimated the mean centre volume velocity for their data base and found a good isotropy with no significant vertical mean velocity.

\section{Discussion}

In their reviews, Granata and Dickey (1991) and Hill et al. (1992) already noticed that adult copepods may possess inertia, and hence may not be true passive tracers. Squires and Yamazaki (1995) have also considered the question of preferential concentration in the framework of copepod studies. However, these papers have been published before several DNS papers could study this issue more thoroughly using for example precise results of contact rates associated with preferential concentration and numerical estimation of the function $G(S t)$. The quite high correction factor given by Eq.(16) can have ecological applications that we discuss here, using available published results on the complex couplings observed between turbulence and plankton abundance.

Consider the optimal turbulent dissipation rate $\epsilon_{\text {opt }}$ corresponding to a particle 


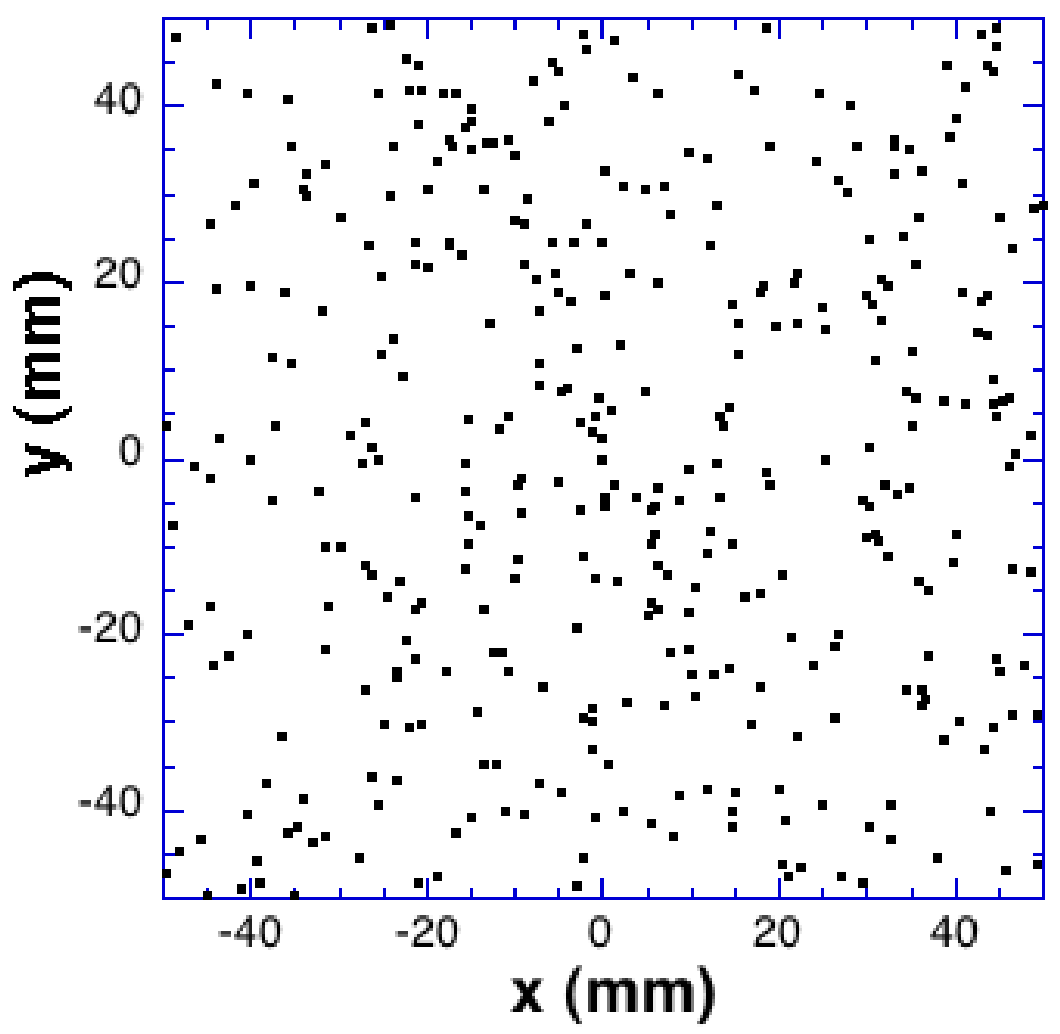

Fig. 2. A 2D projection of particle positions at a given time, as obtained from the experimental study of Ott and Mann (2000).

of diameter $\sigma$. The related Stokes number writes as $S t_{\text {opt }}=0.7 \pm 0.3$. Using Eqs.(5), (7) and (13), we obtain:

$$
\epsilon_{o p t}=\frac{A}{\sigma^{4}}
$$

with $A=\nu^{3}\left(S t_{\text {opt }} / C_{p}\right)^{2}$ for the choice of the Kolmogorov Stokes number, Eq. (5), and $A=\nu^{3}\left(S t_{\text {opt }} / C_{p}\right)^{3}$ for the choice of the $\sigma$-Stokes number, Eq. (13). In both cases, $A$ is a constant depending on the temperature through the viscosity. Equation (17) provides the optimal turbulent dissipation rate for the accumulation of a particle of diameter $\sigma$. Since $G(S t)$ peaks when $S t$ is close to its optimal value, Eq. (17) also provides the turbulence intensity acting as a "resonance" for a given particle size. This is represented in Fig.4, with $\sigma$ in $\mathrm{mm}, \epsilon_{\text {opt }}$ in $\mathrm{cm}^{2} / \mathrm{s}^{3}$ and the constant $A$ estimated for two extreme situations: a lower estimate associated to $S t=0.4$ and high temperature waters $\left(\nu=1.0510^{-6} \mathrm{~m}^{2} / \mathrm{s}\right.$ at $\left.20 \operatorname{deg} C\right)$, and a larger estimate associated to $S t=1$ and low temperature waters $\left(\nu=1.810^{-6} \mathrm{~m}^{2} / \mathrm{s}\right.$ at $\left.0 \operatorname{deg} C\right)$. The corresponding range of values for the constant $A$ are: $A_{\eta} \in[0.6,18]$ and $A_{\sigma} \in[4,300]$ 


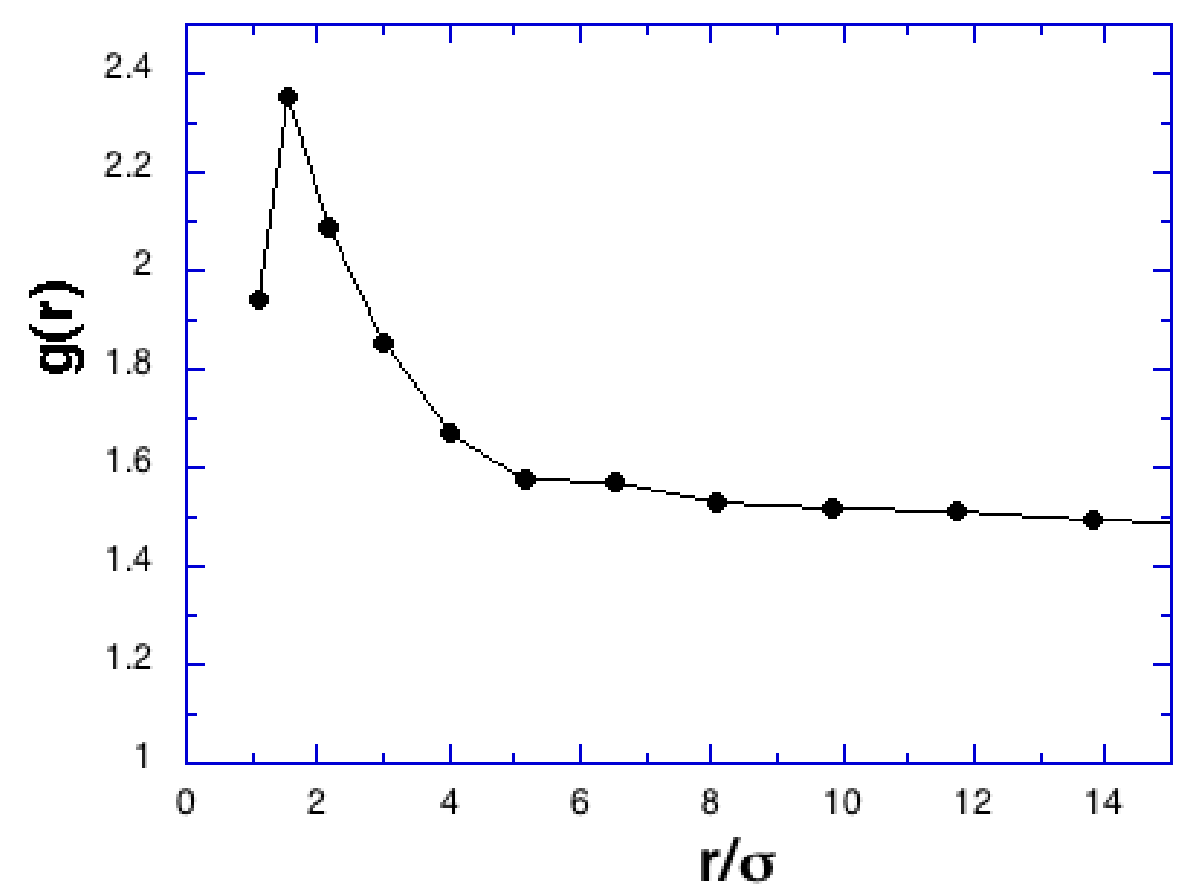

Fig. 3. The function $g(r)$ estimated for the experimental data of Mann et al. (1999). It is computed using available 3D positions of particles An effect of preferential concentration is visible at small distances; the larger value corresponds to twice the diameter of particles.

respectively for the Kolmogorov Stokes number and the $\sigma$-scale Stokes number. There is one order of magnitude difference in the constants $A$, and hence one order of magnitude difference for the optimal dissipation rate associated with the two different choices of Stokes numbers. Figure 4 also shows typical marine environments with their related ranges of dissipation rates taken from (Granata and Dickey, 1991; Kiørboe and Saiz, 1995) and (Druet, 2003): coastal zone, corresponding to $\epsilon$ ranging from $10^{-3}$ to $10^{0} \mathrm{~cm}^{2} / \mathrm{s}^{3}$, oceanic shelf with $\epsilon$ ranging from $10^{-3}$ to $10^{-2} \mathrm{~cm}^{2} / \mathrm{s}^{3}$, and the open ocean with $\epsilon$ being as small as $10^{-6} \mathrm{~cm}^{2} / \mathrm{s}^{3}$. It is interesting to notice from this graph that there is a hierarchy of particle sizes for the different seas. In the coastal zone, only small particles may display preferential concentration, with sizes ranging from 0.8 to $4 \mathrm{~mm}$ : on the oceanic shelf particles range from 2 to $10 \mathrm{~mm}$, and in the open ocean smaller dissipation rates correspond to particle diameters ranging from 1 to $4 \mathrm{~cm}$.

These theoretical results may be interpreted in terms of their consequences for plankton ecology. We first notice that buoyancy free particles with diameters smaller than $0.5 \mathrm{~mm}$ do not seem to be concerned with the preferential concentration effect, since the required turbulence dissipation rate would be too high to be realistic of any marine environment. Only locally, and in very spe- 
cific situations (e.g. breaking waves) the turbulence intensity may reach values high enough to create a preferential concentration effect on such small particles. The same applies to phytoplankton cells. Cells of size such as $50 \mu \mathrm{m}$ may be accumulated only for extreme levels of turbulence such as those induced by strong winds. In addition, this effect might be even more enhanced when plankton blooms are triggered by elevated turbulence intensities (Reigada et al., 2003). However, a local enhancement of copepod concentration may cause a local enhancement of prey capture rate, and in turn increase the small-scale patchiness of the prey. In future work, one may thus also consider the possible influence of copepod behaviour, and the interplay between behavior and turbulence, as initiated in previous works Rothschild and Osborn (1988); Lewis and Pedley (2000).

The preferential concentration effect may also affect planktonic copepods and larval fishes. Indeed, this accumulation effect may be ecologically extremely relevant for copepods as a way to enhance their contact rates with potential sexual partners. However, copepods may have developed behavioral traits to avoid such turbulence intensities since it increases their predation risk, while predators like fish larvae may, in turn, be attracted by the same turbulence intensity as it strongly increases the concentration of their prey. This hypothesis is consistent with the "optimal environmental window" that results in a dome-shaped relation of larval fish ingestion with turbulence intensity (Cury and Roy, 1989; MacKenzie, 2000). This also provides an alternative explanation to the turbulence avoidance strategy proposed by Franks (2001) to explain the enhances in prey ingestion by larval fish within wind-driven mixed-layers.

\section{Conclusion}

We have reviewed here the main results obtained in the field of particle turbulence studies, and their influence on contact rates. These studies have been conducted for inertial particles, mainly in aerosol physics, and correspond to a high density and small particle size relative to the Kolmogorov scale. These studies have shown that the phenomenon of preferential concentration is the largest for Stokes numbers close to 1. They have also provided an important multiplicative correction factor to the encounter rate kernel, which is the radial distribution function at contact $g(\sigma)$. We have considered here the possible application of this effect to copepods. The main difference with aerosols comes from the fact that copepods have a density ratio close to 1, whereas for aerosols it is of the order of $10^{3}$. In order to obtain a similar Stokes number, the particle size must be much larger for copepods than for aerosols. We then assumed here that the numerical results obtained for a density ratio $\beta \gg 1$ are still valid for lighter particles with a density ratio $\beta \approx 1$. This assumption needs experimental validation. Using an experimental data base on particle 


\section{$E_{\text {opt }}\left(\mathrm{cm}^{2} \mathrm{~s}^{-3}\right)$}

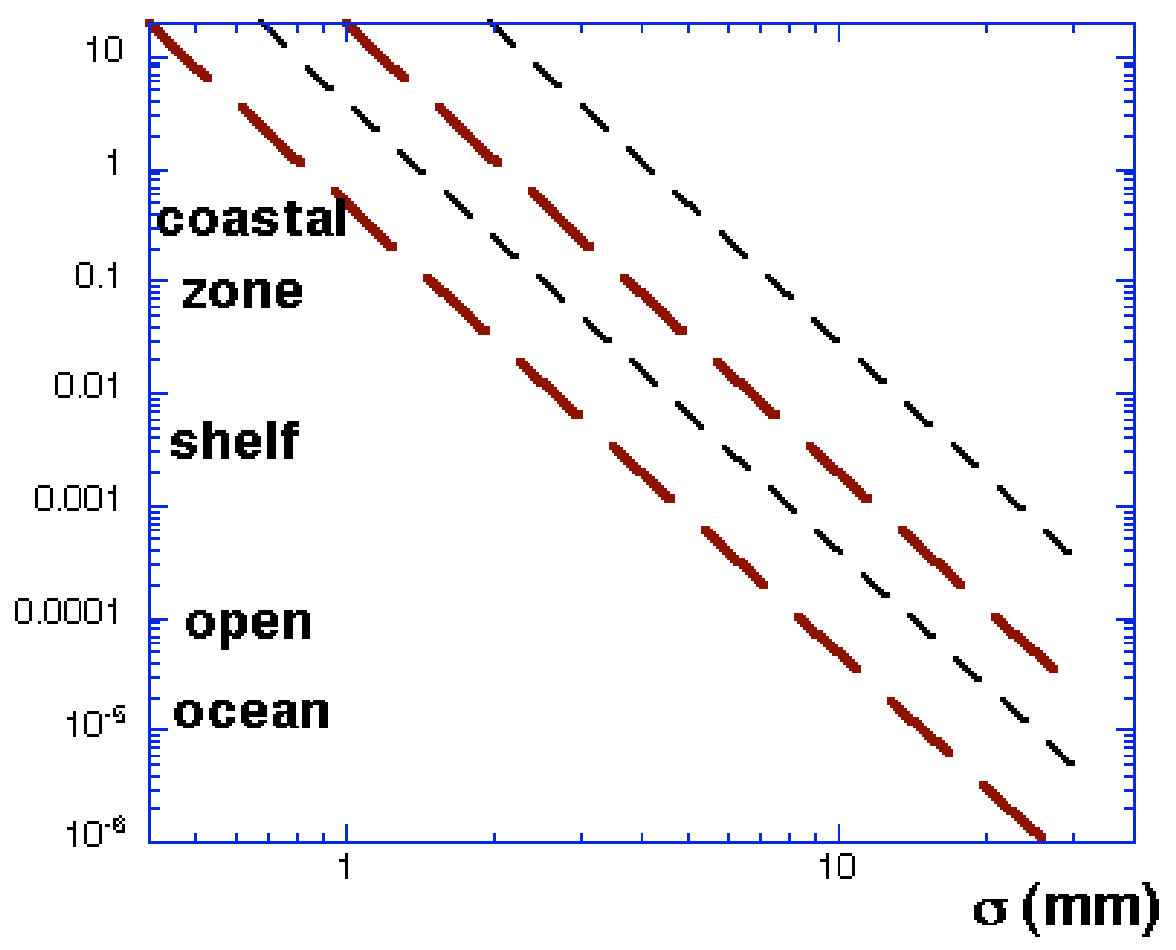

Fig. 4. Estimation of the optimal turbulent dissipation rate corresponding to different values of the particle diameter $\sigma$ : the dotted lines represent the high and low hypothesis for the constant $A$ in Eq.(17), associated to different Stokes number definitions. Typical values of $\epsilon$ in the coastal zone, shelf and open ocean are displayed.

dispersion with buoyancy free particles of size similar to copepods, we have subsequently shown that copepods may also display the effect of preferential concentration when they have a size which is slightly larger than the Kolmogorov scale. Considering that the optimal accumulation effect is obtained for a narrow range of values of the Stokes number $(S t=0.7 \pm 0.3)$, a one-to-one correspondence between optimal particle size and turbulent dissipation rate can be drawn. This shows that for a given flow configuration, associated to a mean turbulent dissipation rate $\epsilon$, there is an optimal plankton size for which there will be a noticeable preferential concentration effect. The shape of the function $G(S t)$ shows that this optimal size may be selected as a resonance effect: it is much larger than 1 only for a short window of $S t$ values. For other $S t$ values, it is close to 1 and there is no accumulation and no increase of the contact rate. Using typical ranges of values of the mean dissipation rate in different marine environments, we have shown that this relation is effective to select large particles ( 1 to $4 \mathrm{~cm}$ ) in the deep seas, and smaller particles $(0.8-4 \mathrm{~mm})$ in surface and coastal waters. This might provide an alternative 
explanation to earlier works suggesting that copepod optimal size was driven by the balance between inertial and viscous forces (Yen , 2000). We have also used this relation to interpret some published results on the different behavior of larval fishes in variable turbulent environments. Finally since the mean abundance of prey may be too low to maintain a population of predators, our results potentially have some implications on zooplankton tophodynamics as predators would critically benefit from dense aggregations of prey. While some behavioral mechanisms have been suggested to explain the formation of patches (Genin et al., 2005), our results show that high intensity turbulence can also create accumulation patches that will be very useful for predators.

\section{Acknowledgements}

We thank professor L.P. Wang for providing the data for Figure 1, and professor J. Mann for sending the dispersion data analysed in section 3, and for interesting email exchanges. We also thank the participants of the WTD 2005 conference for interesting comments and discussions, and especially H. Yamazaki, A. Visser, A. Dominguez, and the organizers of the conference for this very nice event. Patricia "Trish" Lavery kindly improve the language. We acknowledge substantial improvements of the manuscript following the advice of the referees.

\section{References}

Aliseda, A. and Cartellier, A. and Haiinaux, F. and Lasheras, J. C., 2002. Effect of preferential concentration on the settling velocity of heavy particles in homogeneous isotropic turbulence. J. Fluid Mech., 468:77-105.

Babiano A., and Cartwright, J.H.E., and Piro, O., and Provenzale, A., 2000. Dynamics of a small neutrally buoyant sphere in a fluid and targeting Hamiltonian systems. Phys. Rev. Lett., 84:5764-5767.

Bagoien, E., and Kiorboe, T., 2005. Blind dating-mate finding in planktonic copepods. I. Tracking the pheromone trail of Centropages typicus. Mar. Ecol. Prog. Ser., 300:105-115.

Bernal, J. D., Mason, J., and Knight, K. R., 1962. Radial distribution of the random close packing of equal spheres. Nature, 194:956-958.

Bishop, M. and Bruin, C., 1984. The pair correlation function: a probe of molecular order. Am. J. Phys., 52:1106-1108.

Bosse, T. and Kleiser, L. and Meiburg, E., 2006. Small particles in homogeneous turbulence: settling velocity enhancement by two-way coupling. Phys. Fluids, 18:027102. 
Brunk, B. K., Koch, D. L. and Lion, L. W., 1998. Turbulent coagulation of colloidal particles. J. Fluid Mech., 364:81-113.

Buskey, E. J., 1984. Swimming pattern as an indicator of the roles of copepod sensory systems in the recognition of food. Mar. Biol., 79:165-175.

Collins, L. R. and Keswani, A., 2004. Reynolds number scaling of particle clustering in turbulent aerosols. New J. Physics, 6:119.

Cury, P. and Roy, C., 1989. Optimal environmental window and pelagic fish recruitment success in upwelling areas. Can. J. Fish. Aquat. Sci., 46:670-680.

Delichatsios, M. A. and Probstein, R. F., 1975. Coagulation in turbulent flow: theory and experiment. J. Colloid Interface Sci., 51:394-405.

Doall, M.H., Colin, S.P., Strickler, J.R. and Yen, J., 1998. Locating a mate in 3D: the case of Temora longicornis. Phil. Trans. R. Soc. Lond. B, 353:681689.

Druet, C., 2003. The fine structure of marine hydrophysical fields and its influence on the behaviour of plankton: an overview of some experimental and theoretical investigations. Oceanologia, 45:517-555.

Evans, G. T., 1989. The encounter speed of moving predator and prey. J. Plankton Res., 11:415-417.

Falkovich, G., Fouxon, A. and Stepanov, M. G., 2002. Acceleration of rain initiation by cloud turbulence. Nature, 419:151-154.

Fessler, J. R., Kullick, J. D. and Eaton, J. K., 1994. Preferential concentration of heavy particles in a turbulent channel flow. Phys. Fluids., 6:3742-3749.

Franks, P. J. S., 2001. Turbulence avoidance: an alternate explanation of turbulence-enhanced ingestion rates in the field. Limnol. Oceanogr., 46:959963.

Frisch, U., 1995. Turbulence : the legacy of A. N. Kolmogorov. Cambridge University Press, Cambridge.

Gatignol, R., 1983. The Faxen formulas for a rigid particle in an unsteady non-uniform Stokes flow. J. Mec. Theor. Appl., 1:143-154.

Genin, A., Jaffe, J. S., Reef, R., Richter, C. and Franks, P. J., 2005. Swimming against the flow: a mechanism of zooplankton aggregation. Science, 308:860862.

Gerritsen, J. and Strikler, J. R., 1977. Encounter probabilities and community structure in zooplankton : a mathematical model. J. Fish. Res. BOARD CAN., 34:73-82.

Granata, T. C. and Dickey, T. D., 1991. The fluid mechanics of copepod feeding in a turbulent flow: a theoretical approach. Prog. Oceanog., 26:243261.

Hill, P. S., Nowell, A. R. M. and Jumars, P. A., 1992. Encounter rate by turbulent shear of particles similar in diameter to the Kolmogorov scale. J. Mar. Res., 50:643-668.

Jiang, H., Osborn, T. R., and Meneveau, C., 2002. Hydrodynamic interaction between two copepods: a numerical study. J. Plankton Res., 24:235-253.

Jimenez, J., 1997. Oceanic turbulence at millimeter scales. Sci. Mar., 61(Supl. 1):47-56. 
Kiørboe, T. and Saiz, E., 1995. Planktivorous feeding in calm, and turbulent environments with emphasis on copepods. Mar. Ecol. Prog. Ser., 122:135145.

Lenz, P. H., and Yen, J., 1993. Distal setal mechanoreceptors of the first antennae of marine copepods. Bull. Mar. Sci., 53:170-179.

Lewis, D. M. and Pedley, T. J., 2000. Planktonic contact rates in homogeneous isotropic turbulence: theoretical predictions and kinematic simulations. J. theor. Biol., 205:377-408.

Lock, J. A. and Chiu, C.-L., 1994. Correlated light scattering by a dense distribution of condensation droplets on a window pane. Appl. Opt., 33:46634671.

Loth, E., 2000. Numerical approaches for motion of dispersed particles, droplets and bubbles. Prog. En. Comb. Sci., 26:161-223.

MacKenzie, B. R., 2000. Turbulence, larval fish ecology and fisheries recruitment: a review of field studies. Oceanologica Acta, 23:357-375.

Mann, J., Ott, S., and Andersen J. S., 1999. Experimental study of relative, turbulent diffusion. Riso National Laboratory, report Riso-R-1036.

Mann, J., et al., 2005. Turbulent particle flux to a perfectly absorbing surface. J. Fluid Mech., 534:1-21.

Marrasé, C., Costello, J. H., Granata, T. and Strickler J. R., 1990. Grazing in a turbulent environment : energy dissipation, encounter rates, and efficacy of feeding currents in Centropages hamatus. Proc. Natl. Acad. Sci. USA, $87: 1653-1657$.

Maxey, M. R., 1987. The gravitational settling of aerosol particles in homogeneous turbulence and random flow fields. J. Fluid Mech., 174:441-465.

Maxey, M. R. and Riley, J. J., 1983. Equation of motion of a small rigid sphere in a non-uniform flow. Phys. Fluids, 26:883-889.

McCave, I., 1984. Size-spectra and aggregation of suspended particles in the deep ocean. Deep Sea Res., 22:491-502.

Michaelides, E. E., 2003. Hydrodynamic force and heat/mass transfer from particles, bubbles, and drops-The Freeman scholar lecture. J. Fluid Eng., 125:209-238.

Ott, S. and Mann, J., 2000. An experimental investigation of the relative diffusion of particle pairs in three-dimensional turbulent flow. J. Fluid Mech., 422:207-223.

Pan, Y. and Banerjee, S., 1997. Numerical investigation of the effects of large particles on wall-turbulence. Phys. Fluids, 9:3786-3810.

Pathria, R. K., 1972. Statistical Mechanics. Pergamon, New York.

Reade, W. C. and Collins, L. R., 2000. Effect of preferential concentration on turbulent collision rates. Phys. Fluids, 12:2530-2540.

Reigada, R., Hillary, R. M., Bees, M. A., Sancho, J. M., and Sagues, F., 2003. Plankton blooms induced by turbulent flows. Proc. R. Soc. Lond. B, 270:875-880.

Rothschild, B. J. and Osborn, T. R., 1988. Small-scale turbulence and plankton contact rates. J. Plankton Res., 10:465-474. 
Ruiz, J and Macias, D. and Peters, F., 2004. Turbulence increases the average settling velocity of phytoplankton cells. PNAS, 101:17720-17724.

Saffman, P. G. and Turner, J. S., 1956. On the collision of drops in turbulent clouds. J. Fluid Mech., 1:16-30.

Seuront, L., Schmitt, F., and Y. Lagadeuc, 2001. Turbulence intermittency, small-scale phytoplankton patchiness and encounter rates in plankton : where do we go from here? Deep Sea Research I, 48:1199-1215.

Squires, K. D. and Eaton, J. K., 1991. Preferential concentration of particles by turbulence. Phys. Fluids. A, 3:1169-1178.

Squires, K. D. and Yamazaki, H., 1995. Preferential concentration of marine particles in isotropic turbulence. Deep-Sea. Res. I, 42:1989-2004.

Sundaram, S. and Collins, L. R., 1997. Collision statistics in an isotropic particle-laden turbulent suspension. Part 1. Direct numerical simulations. J. Fluid Mech., 335:75-109.

Titelman, J., 2001. Swimming and escape behavior of copepod nauplii : implications for predator-prey interactions among copepods. Mar. Ecol. Prog. Ser., 213:203-213.

Vaillancourt, P. A. and Yau, M. K., 2000. Review of particle-turbulence interactions and consequences for cloud physics. Bull. Am. Meteo. Soc., 81:285298.

Visser, A. W., 2001. Hydromechanical signals in the plankton. Mar. Ecol. Prog. Ser., 222:1-24.

Visser, A. W. and MacKenzie, B. R., 1998. Turbulence-induced contact rates of plankton: the question of scale. Mar. Ecol. Prog. Ser., 166:307-310.

Wang, L. P. and Maxey, M. R., 1993. Settling velocity and concentration distribution of heavy particles in homogeneous isotropic turbulence. J. Fluid Mech., 256:27-68.

Wang, L. P., Wexler, A. S. and Zhou, Y., 1998. Statistical mechanical descriptions of turbulent coagulation. Phys. Fluids, 10:2647-2651.

Wang, L. P., Wexler, A. S. and Zhou, Y., 2000. Statistical mechanical description and modelling of turbulenct collision of inertial particles. J. Fluid Mech., 415:117-153.

Weissburg, M. J., Doall, M. H. and Yen, J., 1998. Following the invisible trail : kinematic analysis of mate-tracking in the copepod Temora longicornis. PhiL. Trans. R. Soc. Lond. B, 353:701-712.

Younge, K., Christenson, C., Bohara, A., Crnkovic, J. and Saulnier, P., 2004. A model for examining the radial distribution function. Am. J. Phys., 72:12471250.

Yen, J., 2000. Life in transition: balancing inertial and viscous forces by planktonic copepods. Biol. Bull., 198:213-224.

Zaichik, L. I., and Simonin, O., and Alipchenkov, V. M., 2006. Collision rates of bidisperse inertial particles in isotropic turbulence. Phys. Fluids, 18:035110.

Zhou, Y., Wexler, A. S. and Wang, L. P., 1998. On the collision rate of small particles in isotropic turbulence. II. Finite inertia case. Phys. Fluids, 10:1206-1216. 
Zhou, Y., Wexler, A. S. and Wang, L. P., 2001. Modelling turbulent collision of bidisperse inertial particles. J. Fluid Mech., 433:77-104. 